\title{
Sind einige SUDEP-Fälle durch Kardiomyopathie verursacht?
}

\begin{abstract}
Neben emotionalem oder physischem Stress sind auch epileptische Anfälle als Trigger für eine TakotsuboKardiomyopathie (TKM) beschrieben. Eine Studie untersucht, ob sich die anfallsbedingte TKM von der durch andere Trigger induzierten TKM unterscheidet.
\end{abstract}

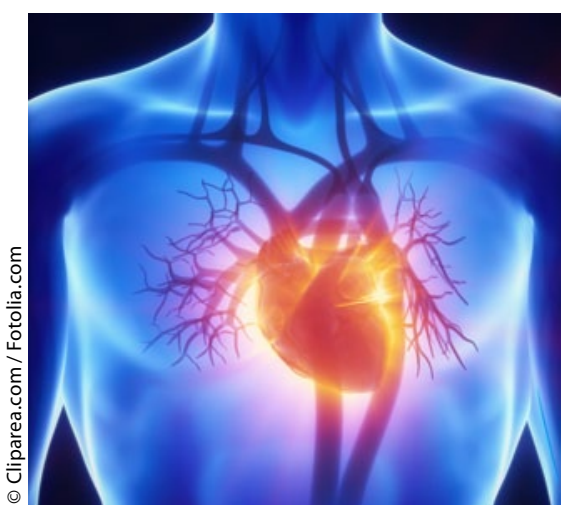

Hinter einem SUDEP kann sich ein kardialer Todesfall verstecken.

ie Takotsubo-Kardiomyopathie (TKM) ist charakterisiert durch Brustschmerzen, Dyspnoe, EKG-Veränderungen wie bei einer akuten koronaren Herzerkrankung sowie transiente Herzwandbewegungsanomalien. Stöllberger et al. verglichen die anfallsassoziierte TKM mit der nicht-anfallsassoziierten Form. Zu den 36 anfallsassoziierten TKM-Fällen (30 Frauen, 6 Männer,
Durchschnittsalter 61,5 Jahre) gehörten solche mit tonisch-klonischen Anfällen $(n=13)$, generalisierten, nicht näher bezeichneten Anfälle ( $\mathrm{n}=5)$, Grand malAnfällen $(\mathrm{n}=2)$, Status epilepticus $(\mathrm{n}=$ 6). Eine Epilepsievorgeschichte hatten 12 Patienten, bei 15 war der TKM-assoziierte Anfall der erste, zu 9 Patienten mit TKM-assoziierten Anfällen gab es keine Angaben. Bei 17 Patienten trat die TKM plötzlich nach dem Anfall auf, bei $9 \mathrm{~Pa}$ tienten in einem Zeitfenster von 1 bis 72 Stunden postiktal und bei 10 Patienten wurde das Intervall nicht erwähnt.

Verglichen mit 974 Patienten mit TKM ohne Anfälle waren die Patienten mit anfallsbedingter TKM jünger (62 vs. 69 Jahre), häufiger männlich (17\% vs. $9 \%)$, hatten seltener Brustschmerzen (6\% vs. $76 \%$ ), häufiger einen kardiogenen Schock (25\% vs. $8 \%$ ) und häufiger wiederholt auftretende TKM (14\% vs. $3 \%)$. Anfallsbedingte TKM manifestierte sich häufiger als plötzliche hämodynamische Entgleisung, die ohne adäquate Hilfe auch zum Tode führen kann. Vermutlich sind einige sogenannte SU-
DEP-Fälle („sudden unexpected death in epilepsy“) durch TKM verursacht.

Kommentar: Solche Arbeiten, die seltene aber wichtige Komorbiditäten betrachten, um Pathomechanismen wie den SUDEP klären zu helfen, sind enorm wichtig für das Verständnis von autonomer Aktivierung in zerebralen Bereichen, die bei Anfällen nicht unbedingt gemessen und monitoriert werden. So werden zwar polygrafisch EKG abgeleitet, aber nur sehr selten Echokardiografien bei SUDEP-gefährdeten Patienten durchgeführt. Ein eigener Fall eines jungen Mannes, der plötzlich unter Videobeobachtung ganz still und nicht anfallsassoziiert verstarb, lenkte das Augenmerk auf diese Studie, nachdem der Pathologe im Obduktionsbericht eine Kardiomyopathie feststellte. Das drei Wochen vor seinem Tod aufgezeichnete EKG war ebenso unauffällig wie die Klinik.

Dr. med. Thomas Mayer

Stöllberger $C$ et al. Seizure-associated Takotsubo cardiomyopathy. Epilepsia 2011; 52 (11): e160-7

\section{Längerfristige Gedächtnisleistungen eingeschränkt}

Patienten mit Epilepsie leiden häufig unter Gedächtnisstörungen. Bisherige Untersuchungen betrafen vor allem die kurzfristigen Behaltensleistungen. Nun zeigt eine Studie, dass zuvor erlernte Inhalte häufig im längerfristigen Verlauf vergessen werden.

D as Konzept des akzelerierten Vergessens (,accelerated long-term forgetting", ALF) geht davon aus, dass sich ein pathologisches Vergessen zuvor erlernter Inhalte häufig noch nicht im kurzfristigen zeitlichen Rahmen, sondern erst im längerfristigen Verlauf über Tage oder Wochen hinweg zeigt. Bisher standen überwiegend die kurzfristigen Behaltensleistungen im Fokus der neuropsychologischen Untersuchungen. Fitzgerald et al. haben nun die Einflussfaktoren auf die längerfristigen Behaltensleistungen von Epilep- siepatienten untersucht. Hierzu wurden den Patienten eine Wortliste, sowie eine Liste mit abstrakten Zeichnungen zum Lernen vorgegeben und nach 30 Minuten, einem Tag und vier Tagen erneut abgefragt. Die Besonderheit der Studie: Alle Patienten wurden über die gesamte Untersuchungsdauer hinweg mit einem mobilen LangzeitEEG ausgestattet. Dieses Vorgehen ermöglichte es, Anfälle, subklinische Anfallsmuster und weitere epilepsietypische Auffälligkeiten exakt zu erfassen und die Schlafarchitektur wie
Schlafeffizienz zu bewerten. Daneben wurden weitere epilepsieassoziierte Variablen (Medikation, MRT-Befunde, Epilepsieformen, Dauer der Erkrankung, Depression) in Bezug zur Gedächtnisleistung gesetzt.

Eingeschlossen in die Studie wurden 33 Epilepsiepatienten in drei Gruppen (normales EEG $/ \mathrm{n}=18$, Patienten mit fokalen $/ \mathrm{n}=10$ und generalisierten Entladungen $/ \mathrm{n}=5$ ) sowie 15 Kontrollprobanden ohne Epilepsie. Während sich die vier Gruppen im 30 Minuten verzögerten Gedächtnisabruf nicht unterschieden, 